\title{
UNA DEMOSTRACIÓN DEL TEOREMA DE GEARHART
}

\author{
Yolanda Santiago Ayala ${ }^{1}$
}

\begin{abstract}
Resumen: Usando la noción del Tipo del Semigrupo, Solución periódica de una ecuación no homogénea, los Principios fundamentales del análisis funcional, y fuertemente las nociones de Teoría espectral, damos una prueba del famoso e importante resultado de Gearhart, acerca de la estabilidad exponencial de un Semigrupo, introducido en Liu-Zheng [1].
\end{abstract}

Palabras clave: Radio espectral. El tipo del semigrupo. Semigrupo exponencialmente estable. Solución periódica de una ecuación no homogénea. Teorema de Gearhart

\section{A PROOF OF THE GEARHART'S THEOREM}

\begin{abstract}
Using the notion of semigroup type, periodic solution of a nonhomogeneous equation, the fundamental principles of functional analysis, and strongly the notions of spectral theory, we give a proof of the famous and important result of Gearhart, about exponential stability of a semigroup, introduced in LiuZheng [1].
\end{abstract}

Keywords: Spectral radius. The type of semigroup. Exponentially stable semigroup. Periodic solution of non-homogeneous equation. The Gearthart's theorem.

\section{Introducción}

Para determinar si una solución decae exponencialmente frecuentemente se usaba el método de Prüs [3] y desigualdades integrales con propiedades de semigrupo. Ahora, queremos enfatizar que tenemos la otra opción que es el Teorema de Gearhart, introducido por Liu-Zheng [1].

Este famoso Teorema de Gearhart nos da la caracterización de la estabilidad exponencial de un semigrupo, i.e., condiciones necesarias y suficientes para la estabilidad exponencial en términos del resolvente del generador infinitesimal del Semigrupo asociado al sistema.

Nuestro principal interés es enunciar y probar el Teorema de Gearhart, para eso introducimos y probamos dos caracterizaciones de $1 \in \rho(S(1))$, dando una completa prueba.

Nuestro artículo está organizado como sigue. En la sección 2, enunciamos y probamos dos resultados importantes, que usaremos para probar el Teorema de Gearhart, i.e., dos caracterizaciones de $1 \in \rho(S(1))$. La primera caracterización en conexión con la existencia y unicidad de solución periódica de una ecuación no homogénea y la segunda en términos de la acotación del Operador Resolvente: $R(2 k \pi i, A), \forall k \in \mathbb{Z}$.

En la sección 3, enunciamos y probamos el Teorema de Gearhart.

En la sección 4, damos algunos comentarios de ejemplos de aplicación.

\footnotetext{
${ }^{1}$ UNMSM, Facultad de Ciencias Matemáticas, e-mail: yssantiago@gmail.com
} 


\section{Principales Resultados}

Definición 2.1 Sea A el generador infinitesimal de un semigrupo $C_{o}(S(t))_{t \geq 0}$. Diremos que $w_{o}(A)$ es el tipo del semigrupo generado por $A$ si existe el número

$$
w_{o}(A):=\lim _{t \rightarrow+\infty} \frac{\ln \|S(t)\|}{t}=\inf _{t>0} \frac{\ln \|S(t)\|}{t} .
$$

Observación 2.2 Sea $s>0$ y $A$ el generador infinitesimal de un semigrupo $C_{o}(S(t))_{t \geq 0}$. Entonces $w_{o}(s A)=s w_{o}(A)$.

Lema 2.3 Sea A el generador infinitesimal de un semigrupo de clase $C_{o}(S(T))_{t \geq 0}$. Entonces el radio espectral de $S(t)$ es igual a $e^{t w_{o}(A)}$, para $t>0$. i.e.,

$$
r_{\sigma}(S(t))=e^{t w_{o}(A)}, \forall t>0 .
$$

Teorema 2.4 Sea $X$ un espacio de Banach, $f \in C([0,1] ; X)$ y A el generador infinitesimal de un semigrupo $C_{o}(S(t))_{t \geq 0}$. La ecuación

$$
u^{\prime}(t)=A u(t)+f(t), \quad t \in[0,1]
$$

posee una única solución periódica, de periodo 1 , si y solamente si $1 \in \rho(S(1))$.

Demostración. Si $1 \in \rho(S(1))$ probaremos que la ecuación (1) posee una única solución periódica.

Existencia de solución

Supongamos que $u(t)$ sea una solución de (1), entonces

$$
\frac{d}{d t}[S(-t) u(t)]=S(-t) f(t)
$$

Integrando de 0 a $t$ tenemos

$$
\left.\left.\int_{0}^{t} \frac{d}{d s}\right] S(-s) u(s)\right] d s=\int_{0}^{t} S(-s) f(s) d s .
$$

Luego, $S(-t) u(t)-\underbrace{S(0)}_{=I} u(0)=\int_{0}^{t} S(-s) f(s) d s$, aplicando $S(t)$ tenemos

$$
\begin{aligned}
u(t)-S(t) u(0) & =S(t) \int_{0}^{t} S(-s) f(s) d s \\
& =\int_{0}^{t} S(t) S(-s) f(s) d s \\
& =\int_{0}^{t} S(t-s) f(s) d s
\end{aligned}
$$

i.e., $u(t)$ tendría la forma:

$$
u(t)=\underbrace{S(t) u(0)}_{z(t):=}+\int_{0}^{t} S(t-s) f(s) d s
$$

donde $z$ es solución de la ecuación homogénea asociada a (1), esto es, $z^{\prime}(t)=A z(t), z(0)=u(0)$, y como queremos que $u(0)=u(1), u$ debe satisfacer

$$
u(0)=u(1)=S(1) u(0)+\int_{0}^{1} S(1-s) f(s) d s,
$$


es decir

$$
[I-S(1)] u(0)=\int_{0}^{1} S(1-s) f(s) d s .
$$

Como $1 \in \rho(S(1))$ entonces existe $[I-S(1)]^{-1}$ y así $u(0)$ sería

$$
u(0)=[I-S(1)]^{-1} \int_{0}^{1} S(1-s) f(s) d s .
$$

Luego

$$
\begin{gathered}
u(t)=S(t) u(0)+\int_{0}^{t} S(t-s) f(s) d s \text { con } \\
u(0)=[I-S(1)]^{-1} \int_{0}^{1} S(1-s) f(s) d s
\end{gathered}
$$

es una solución periódica de la ecuación (1). A seguir veremos que es única.

Unicidad de solución

Sean $u_{i}(t)$ para $i=1,2$ soluciones periódicas de la ecuación (1), luego satisfacen

$$
u_{i}^{\prime}(t)=A u_{i}(t)+f(t), u_{i}(0)=u_{i}(1) \text { para } i=1,2 .
$$

y $u_{i}(t)$ tienen la forma

$$
u_{i}(t)=S(t) u_{i}(0)+\int_{0}^{t} S(t-s) f(s) d s
$$

con $u_{1}(0)=u_{2}(0)$, desde que

$$
u_{i}(0)=[I-S(1)]^{-1} \int_{0}^{1} S(1-s) f(s) d s \text { para } i=1,2 .
$$

Luego,

$$
u_{1}(t)-u_{2}(t)=S(t)[\underbrace{u_{1}(0)-u_{2}(0)}_{=0}]=0
$$

Recíprocamente, supongamos que la ecuación (1) tiene una única solución periódica, probaremos que $1 \in \rho(S(1))$, es decir que existe $[I-S(1)]^{-1} \in L(X)$.

$I-S(1)$ es inyectiva

Procedemos por el absurdo. Supongamos que

$$
\operatorname{Ker}(I-S(1)) \neq\{0\},
$$

i.e., $\exists x_{0} \in \operatorname{Ker}(I-S(1))$ tal que $x_{0} \neq 0$, es decir $x_{0} \neq 0$ y

$$
x_{0}-S(1) x_{0}=0 .
$$

Definimos

$$
u(t):=S(t) x_{0}
$$

Luego, por (4) tenemos

$$
u(1)=S(1) x_{0}=\underbrace{x_{0}}_{\neq 0}=S(0) x_{0}=u(0)
$$

y

$$
u(t+1)=S(t+1) x_{0}=S(t) \underbrace{S(1) x_{0}}=S(t) x_{0}=u(t),
$$

i.e., $u(1)=u(0), u$ es periódica, de periodo 1 y $u \not \equiv 0\left(u(1)=x_{0} \neq 0\right)$.

También, se tiene que $u$ satisface

$$
u^{\prime}(t)-A u=A S(t) x_{0}-A S(t) x_{0}=0 .
$$

Luego existe $u \not \equiv 0$ y $v \equiv 0$ soluciones periódicas de (5) con $f \equiv 0$, lo cual es absurdo, por hipótesis. Así, $I-S(1)$ es inyectiva. 
$I-S(1): X \longrightarrow X$ es sobreyectiva

$\overline{\text { Sea } x \in X \text {, probaremos que existe } y} \in X$ tal que $(I-S(1)) y=x$.

Definimos,

$$
\begin{aligned}
K: C([0,1], X) & \rightarrow C([0,1], X) \\
f & \rightarrow K f:=u
\end{aligned}
$$

donde $u \equiv u(t)$ es la única solución periódica de la ecuación (1) respecto a $f$, la cual existe por hipótesis.

Se observa fácilmente que $K$ es un operador lineal y cerrado.

$\underline{K \text { es continuo: }}$

Como $K$ es un operador lineal cerrado, usando el Teorema del gráfico cerrado tenemos que $K$ es un operador lineal continuo.

Definimos el operador

$$
\begin{aligned}
T: X & \rightarrow X \\
x & \rightarrow T x:=(K f)(0)=u(0)
\end{aligned}
$$

donde como $f(t)$ estamos considerando $S(t) x$, i.e., $f(t):=S(t) x, f(0)=x$, y $u$ es la única solución periódica de (1) respecto a esta $f$.

Observamos que $\underline{T \text { es continua, }}$, desde que $K, \mathcal{M}$, y $\mathcal{N}$ lo son, donde $\mathcal{M}(x)=f, \mathcal{M}: X \rightarrow$ $C([0,1], X)$ y $\mathcal{N}(g)=g(0), \mathcal{N}: C([0,1], X) \rightarrow \mathbb{R}$, i.e., $T=\mathcal{N} \circ K \circ \mathcal{M}$.

De la expresión de $u(2)$, en particular para $t=1$, tenemos

$$
\begin{aligned}
\underbrace{u(0)}_{=T x}=u(1) & =S(1) u(0)+\int_{0}^{1} S(1-s) f(s) d s \\
& =S(1) u(0)+\int_{0}^{1} S(1-s) S(s) x d s \\
& =S(1) u(0)+\int_{0}^{1} S(1) x d s \\
& =S(1) \underbrace{u(0)}_{=T x}+S(1) x \pm x
\end{aligned}
$$

esto es,

$$
[I-S(1)](\underbrace{T x+x}_{y:=})=x
$$

o

$$
[I-S(1)][T+I] x=x
$$

i.e., $\exists y=T x+x \in X$ tal que $[I-S(1)] y=x$.

Luego $I-S(1)$ es sobreyectiva.

Así, $I-S(1)$ es biyectiva y como es continua, podemos aplicar el Teorema de la aplicación abierta y concluir que $I-S(1)$ es un homeomorfismo, es decir que $[I-S(1)]^{-1}$ es continuo. Por lo tanto, $1 \in \rho(S(1))$ y además de $(7)$ tenemos $[I-S(1)]^{-1}=T+I$.

Teorema 2.5 Sea A el generador infinitesimal de un semigrupo $C_{o}(S(t))_{t \geq 0}$. Entonces $1 \in$ $\rho(S(1))$ si y solamente si,

$$
2 k \pi i \in \rho(A), \quad \forall k \in Z
$$

y

$$
\sup _{k \in Z}\left\|(2 k \pi i I-A)^{-1}\right\|<\infty .
$$


Demostración. Tenemos

$$
\begin{aligned}
S(1) e^{-2 k \pi i I} x-S(0) x & =\int_{0}^{1} \frac{d}{d s}\left\{S(s) e^{-2 k \pi i I s}\right\} x d s \\
& =\int_{0}^{1}[A-2 k \pi i I] S(s) e^{-2 k \pi i I s} x d s .
\end{aligned}
$$

Como $S(1) \underbrace{e^{-2 k \pi i I}}_{=I}=S(1)$, obtenemos

$$
S(1) x-x=\int_{0}^{1}[A-2 k \pi i I] S(s) e^{-2 k \pi i I s} x d s
$$

Luego,

$$
[S(1)-I] x=[A-2 k \pi i I] \int_{0}^{1} S(s) e^{-2 k \pi i I s} x d s .
$$

Así, desde que $1 \in \rho(S(1))$, obtenemos conmutando

$$
\begin{aligned}
x & =[S(1)-I]^{-1}[A-2 k \pi i I] \int_{0}^{1} S(s) e^{-2 k \pi i I s} x d s \\
& =[A-2 k \pi i I] \underbrace{[S(1)-I]^{-1} \int_{0}^{1} S(s) e^{-2 k \pi i I s} x d s}_{B x:=} \\
& =[A-2 k \pi i I] B x .
\end{aligned}
$$

También, conmutando tenemos

$$
\begin{aligned}
x & =[S(1)-I]^{-1} \int_{0}^{1}[A-2 k \pi i I] S(s) e^{-2 k \pi i I s} x d s \\
& =[S(1)-I]^{-1} \int_{0}^{1} S(s) e^{-2 k \pi i I s} \underbrace{[A-2 k \pi i I] x} d s \\
& =B([A-2 k \pi i I] x) .
\end{aligned}
$$

De las igualdades (8) y (9), concluimos que existe $[A-2 k \pi i I]^{-1}=B$ y que

$$
\begin{aligned}
\left\|[A-2 k \pi i I]^{-1} x\right\| & =\|B x\| \\
& =\left\|[S(1)-I]^{-1} \int_{0}^{1} S(s) e^{-2 k \pi i I s} x d s\right\| \\
& \leq M\|\int_{0}^{1} \underbrace{S(s)}_{\|S(s)\| \leq M_{1}} e^{-2 k \pi i I s} x d s\| \\
& \leq M_{2}\|x\| .
\end{aligned}
$$

Finalmente, hemos probado que $2 k \pi i \in \rho(A) \mathrm{y} \mathrm{sup}_{k}\left\|[A-2 k \pi i I]^{-1}\right\| \leq M_{2}$.

Recíprocamente, para probar que $1 \in \rho(S(1))$, usaremos el Teorema anterior. Así, basta mostrar que la ecuación (1) posee una única solución periódica.

Sea $u$ una solución de la ecuación (1), es decir satisface $u^{\prime}(t)=A u(t)+f(t)$ con $u(0)=u(1)$.

Definimos $\forall k \in \mathbb{Z}$,

$$
\begin{aligned}
u_{k} & :=\int_{0}^{1} u(s) e^{-2 k \pi i s} d s \\
f_{k} & :=\int_{0}^{1} f(s) e^{-2 k \pi i s} d s
\end{aligned}
$$




\section{Afirmamos:}

$$
u_{k}=[2 k \pi i I-A]^{-1} f_{k}
$$

Unicidad de Solución

Sea $v$ otra solución de (1), entonces por (10) tenemos que

$$
v_{k}=[2 k \pi i I-A]^{-1} f_{k},
$$

es decir, $v_{k}=u_{k} \forall k$. Luego, por Series de Fourier, $v=u$, es decir, la solución de (1) es única.

underlineExistencia de Solución

Definimos

$$
\begin{aligned}
U_{N}(t) & :=\sum_{k=-N}^{N} u_{k} e^{2 k \pi i t} \\
F_{N}(t) & :=\sum_{k=-N}^{N} f_{k} e^{2 k \pi i t}
\end{aligned}
$$

\section{Observación 2.6}

$$
f=\sum_{-\infty}^{\infty} f_{k} e^{2 k \pi i t}=\lim _{N \rightarrow+\infty} F_{N} \text { en } L^{2}(0,1)
$$

$y$

$$
\|f\|_{L^{2}(0,1)}^{2}=\sum_{-\infty}^{+\infty}\left\|f_{k}\right\|^{2} .
$$

Afirmamos: $U_{N}$ satisface (1) con $F_{N}$. Es decir, se verifica

$$
\mid \begin{aligned}
& \frac{d}{d t} U_{N}=A U_{N}+F_{N} \\
& U_{N}(0)=U_{N}(1) .
\end{aligned}
$$

En efecto, $U_{N}(0)=\sum_{k=-N}^{N} u_{k}=U_{N}(1)$. También, usando (10) tenemos

$$
\begin{aligned}
\frac{d}{d t} U_{N}(t)-A U_{N}(t) & =\sum_{k=-N}^{N}(2 k \pi i) u_{k} e^{2 k \pi i t}-\sum_{k=-N}^{N} A u_{k} e^{2 k \pi i t} \\
& =\sum_{k=-N}^{N} \underbrace{[2 k \pi i I-A] u_{k}} e^{2 k \pi i t} \\
& =\sum_{k=-N}^{N} f_{k} e^{2 k \pi i t}=F_{N}(t) .
\end{aligned}
$$

La ecuación (11) implica

$$
U_{N}(t)=S(t) U_{N}(0)+\int_{0}^{t} S(t-s) F_{N}(s) d s .
$$

Considerando $t=1$ en (12) tenemos

$$
\underbrace{U_{N}(1)}_{=U_{N}(0)}=S(1) U_{N}(0)+\int_{0}^{1} S(1-s) F_{N}(s) d s,
$$

es decir,

$$
(I-S(1)) U_{N}(0)=\int_{0}^{1} S(1-s) F_{N}(s) d s .
$$




\section{Afirmamos:}

$$
(I-S(1)) U_{N}(0) \text { es convergente en } X \text {. }
$$

En efecto, basta mostrar que es de Cauchy,

$$
\begin{aligned}
\left\|\int_{0}^{1} S(1-s)\left\{F_{N}(s)-F_{L}(s)\right\} d s\right\| \leq & \int_{0}^{1}\left\|S(1-s)\left\{F_{N}(s)-F_{L}(s)\right\}\right\| d s \\
& =\int_{0}^{1}\|S(1-s)\|\left\|F_{N}(s)-F_{L}(s)\right\| d s \\
\leq & M \int_{0}^{1}\left\|F_{N}(s)-F_{L}(s)\right\| d s \\
\leq & M\left[\int_{0}^{1}\left\|F_{N}(s)-F_{L}(s)\right\|^{2} d s\right]^{\frac{1}{2}} \\
= & M\left\|F_{N}-F_{L}\right\|_{L^{2}(0,1)}^{\longrightarrow}
\end{aligned}
$$

cuando $N, L \rightarrow+\infty$.

Así, la sucesión $(I-S(1)) U_{N}(0)$ es de Cauchy en $X$, y como $X$ es un espacio de Banach, la sucesión $(I-S(1)) U_{N}(0)$ es convergente en $X$.

Por otro lado, aplicando $S(1-t)$ a la igualdad (12) tenemos

$$
S(1-t) U_{N}(t)=S(1) U_{N}(0)+S(1-t) \int_{0}^{t} S(t-s) F_{N}(s) d s
$$

es decir,

$$
S(1) U_{N}(0)=S(1-t) U_{N}(t)-S(1-t) \int_{0}^{t} S(t-s) F_{N}(s) d s
$$

e integrando esta expresión de 0 a 1 conseguimos

$$
S(1) U_{N}(0)=\int_{0}^{1} S(1-t) U_{N}(t) d t-\int_{0}^{1} S(1-t) \int_{0}^{t} S(t-s) F_{N}(s) d s d t
$$

\section{Afirmamos:}


En efecto, sea $L<N$,

$$
\begin{aligned}
& \left\|\int_{0}^{1} S(1-s)\left\{U_{N}(s)-U_{L}(s)\right\} d s\right\| \leq \int_{0}^{1}\left\|S(1-s)\left\{U_{N}(s)-U_{L}(s)\right\}\right\| d s \\
& =\int_{0}^{1}\|S(1-s)\|\left\|U_{N}(s)-U_{L}(s)\right\| d s \\
& \leq M \int_{0}^{1}\left\|U_{N}(s)-U_{L}(s)\right\| d s \\
& \leq M \int_{0}^{1}\left\|\sum_{L}^{N} u_{k} e^{2 k \pi i s}+\sum_{-N}^{-L-1} u_{k} e^{2 k \pi i s}\right\| d s \\
& \leq M \int_{0}^{1}\left\{\sum_{L}^{N}\left\|u_{k}\right\|+\sum_{-N}^{-L-1}\left\|u_{k}\right\|\right\} d s \\
& \leq M\left\{\sum_{L}^{N}\left\|[2 k \pi i I-A]^{-1}\right\|\left\|f_{k}\right\|\right. \\
& \left.+\sum_{-N}^{-L-1}\left\|[2 k \pi i I-A]^{-1}\right\|\left\|f_{k}\right\|\right\} \\
& \leq M M_{2}\left\{\sum_{L}^{N}\left\|f_{k}\right\|+\sum_{-N}^{-L-1}\left\|f_{k}\right\|\right\} \\
& \leq M M_{2}\left\{\sum_{L}^{N}\left\|f_{k}\right\|^{2}+\sum_{-N}^{-L-1}\left\|f_{k}\right\|^{2}\right\}^{2} \\
& \leq M M_{2}\left\{\sum_{-N}^{N}\left\|f_{k}\right\|^{2}-\sum_{-L}^{L}\left\|f_{k}\right\|^{2}\right\}^{2} \\
& \longrightarrow 0
\end{aligned}
$$

cuando $N, L \rightarrow+\infty$. Luego la sucesión es de Cauchy en $X$ por lo tanto es convergente en $X$. Ahora nos falta ver que el segundo término de la sucesión $s(1) U_{N}(0)$ es convergente, para eso hacemos lo siguiente

$$
\begin{aligned}
\left\|\int_{0}^{1} S(1-t) \int_{0}^{t} S(t-s)\left\{F_{N}(s)-F_{L}(s)\right\} d s d t\right\| & \leq \int_{0}^{1}\|S(1-t)\| \int_{0}^{t}\|S(t-s)\|\left\|F_{N}(s)-F_{L}(s)\right\| d s d t \\
& \leq M^{2} \int_{0}^{1}\left\|F_{N}(s)-F_{L}(s)\right\| d s \\
& \leq M^{2}\left[\int_{0}^{1}\left\|F_{N}(s)-F_{L}(s)\right\|^{2} d s\right]^{\frac{1}{2}} \\
& \leq M^{2}\left\|F_{N}-F_{L}\right\|_{L^{2}(0,1)} \\
& \longrightarrow 0
\end{aligned}
$$

cuando, $N, L \rightarrow+\infty$.

Como

$$
U_{N}(0)=(I-S(1)) U_{N}(0)+S(1) U_{N}(0),
$$

de (14) y (16) tenemos que $U_{N}(0)$ es convergente en $X$, i.e., $\exists u_{0} \in X$ tal que $U_{N}(0) \rightarrow u_{0}$ y como $U_{N}(0)=U_{N}(1)$ se tiene que $U_{N}(1) \rightarrow u_{0}$.

Afirmamos:

$$
S(t) U_{N}(0) \text { converge en } L^{2}(0,1) \text {. }
$$


y mejor aún

$$
S(t) U_{N}(0) \text { converge a } S(t) u_{0} \text { en } L^{2}(0,1) .
$$

En efecto, basta mostrar que $S(t) U_{N}(0)$ es de Cauchy en $L^{2}(0,1)$. Hacemos,

$$
\begin{aligned}
\int_{0}^{1}\left\|S(t)\left\{U_{N}(0)-U_{L}(0)\right\}\right\|_{X}^{2} d t & \leq \int_{0}^{1} \underbrace{\|S(t)\|^{2}}_{\leq M^{2}}\left\|U_{N}(0)-U_{L}(0)\right\|_{X}^{2} d t \\
\leq & M^{2} \int_{0}^{1}\left\|U_{N}(0)-U_{L}(0)\right\|_{X}^{2} d t \\
& =M^{2}\left\|U_{N}(0)-U_{L}(0)\right\|_{X}^{2} \\
& \longrightarrow 0
\end{aligned}
$$

cuando $N, L \rightarrow+\infty$.

Siguiendo los cálculos como arriba tenemos que

$$
\int_{0}^{1}\left\|S(t)\left\{U_{N}(0)-u_{0}\right\}\right\|_{X}^{2} d t \leq M^{2}\left\|U_{N}(0)-u_{0}\right\|_{X}^{2} \longrightarrow 0
$$

cuando $N \rightarrow+\infty$.

\section{Afirmamos:}

$$
\int_{0}^{t} S(t-s) F_{N}(s) d s \text { converge en } L^{2}(0,1)
$$

y mejor aún

$$
\int_{0}^{t} S(t-s) F_{N}(s) d s \text { converge a } \int_{0}^{t} S(t-s) f(s) d s \text { en } L^{2}(0,1)
$$

En efecto,

$$
\begin{aligned}
\int_{0}^{1} & \left\|\int_{0}^{t} S(t-s)\left\{F_{N}(s)-F_{L}(s)\right\} d s\right\|^{2} d t \\
& \leq \int_{0}^{1}\left\{\int_{0}^{t}\|S(t-s)\|\left\|F_{N}(s)-F_{L}(s)\right\| d s\right\}^{2} d t \\
& \leq M^{2} \int_{0}^{1}\left\{\int_{0}^{t}\left\|F_{N}(s)-F_{L}(s)\right\| d s\right\}^{2} d t \\
& \leq M^{2}\left\{\int_{0}^{1}\left\|F_{N}(s)-F_{L}(s)\right\| d s\right\}^{2} \\
& \leq M^{2}\left\{\int_{0}^{1}\left\|F_{N}(s)-F_{L}(s)\right\|^{2} d s\right\} \\
& =M^{2}\left\|F_{N}-F_{L}\right\|_{L^{2}(0,1)}^{2} \\
& \longrightarrow 0
\end{aligned}
$$

cuando $N, L \rightarrow+\infty$.

Siguiendo los cálculos como arriba conseguimos,

$$
\left.\int_{0}^{1} \| \int_{0}^{t} S(t-s)\left\{F_{N}(s)-f(s)\right)\right\} d s\left\|^{2} d t \leq M^{2}\right\| F_{N}-f \|_{L^{2}(0,1)}^{2} \rightarrow 0
$$

cuando $N \rightarrow+\infty$. 
Luego, $U_{N}$ converge a $w$ en $L^{2}(0,1)$ donde

$$
w(t)=S(t) u_{0}+\int_{0}^{t} S(t-s) f(s) d s .
$$

Ya vimos que $w(1)=w(0)=u_{0}$, y se prueba que $w$ es solución de (1) para $f$. En efecto, para eso introducimos un elemental e importante resultado de Cálculo,

Observación 2.7 $\frac{d}{d t}\left\{\int_{0}^{t} g(t, s) d s\right\}=g(t, t)+\int_{0}^{t} \frac{d}{d t} g(t, s) d s$.

Ahora, procedemos a hacer los cálculos

\section{Observación 2.8}

$$
\begin{aligned}
\frac{d}{d t}\left\{\int_{0}^{t} S(t-s) f(s) d s\right\} & =S(0) f(t)+\int_{0}^{t} \frac{d}{d t} S(t-s) f(s) d s \\
& =f(t)+\int_{0}^{t} A S(t-s) f(s) d s \\
& =f(t)+A \int_{0}^{t} S(t-s) f(s) d s .
\end{aligned}
$$

Observación 2.9 Sea $u(t):=S(t) u_{0}+\int_{0}^{t} S(t-s) f(s) d s$, entonces $u$ satisface $u^{\prime}(t)=$ $A u(t)+f(t)$.

Luego, por unicidad $w=u$. Esto es, existe la solución de (1), que se expresa por

$$
u(t)=S(t) u_{0}+\int_{0}^{t} S(t-s) f(s) d s .
$$

Corolario 2.10 Sea $A$ el generador infinitesimal de un semigrupo $C_{o}$. Entonces $e^{\lambda t} \in \rho(S(t))$ si y solamente si

$$
\lambda+\frac{2 k \pi i}{t} \in \rho(A) \quad y \quad\left\|\left(\left(\lambda+\frac{2 k \pi i}{t}\right) I-A\right)^{-1}\right\|<\infty, \forall k \in Z .
$$

Observación 2.11 Este corolario nos dice que: $1 \in \rho(S(t))$ si y solamente si

$$
\frac{1}{t} L_{\mu} \subset \rho(A) \quad \text { y } \sup _{\lambda \in \frac{1}{t} L_{\mu}}\left\|(\lambda I-A)^{-1}\right\|<\infty .
$$

\section{El Teorema de Gearhart}

A seguir enunciamos y probamos el importante resultado de caracterización de un Semigrupo $C_{o}$ exponencialmente estable.

Teorema 3.1 (Gearhart) Sea $(S(t))_{t \geq 0}$ un semigrupo $C_{o}$ de contracciones en un espacio de Hilbert. Entonces, $(S(t))_{t \geq 0}$ es exponencialmente estable (esto es, $\exists M \geq 1, \mu>0$ tal que $\left.\|S(t)\| \leq M e^{-\mu t}, \forall t \geq 0\right)$ si y solamente si

a) $\rho(A) \supset i \mathbb{R}:=\{i \beta, \beta \in \mathbb{R}\}$

b) $\lim \sup _{|\beta| \rightarrow \infty}\left\|(i \beta I-A)^{-1}\right\|<\infty$. 
Demostración. Desde que $\|S(t)\|<|\lambda|$ y por el Teorema del Inverso tenemos que existe

$$
\begin{aligned}
(\lambda I-S(t))^{-1} & =\left(\lambda\left(I-\lambda^{-1} S(t)\right)\right)^{-1} \\
& =\lambda^{-1} \underbrace{\left(I-\lambda^{-1} S(t)\right)^{-1}}_{\exists} \\
& =\lambda^{-1}\left(I+\frac{S(t)}{\lambda}+\frac{S(t)^{2}}{\lambda^{2}}+\ldots\right) .
\end{aligned}
$$

Esto es, si $\|S(t)\|<|\lambda|$, entonces $\lambda \in \rho(S(t))$.

Luego, si $\lambda \in \sigma(S(t))$ entonces $\|S(t)\| \geq|\lambda|$.

Como $(S(t))_{t \geq 0}$ es un semigrupo $C_{o}$ de contracciones, tenemos que $\|S(t)\| \leq 1$, luego

$$
\sigma(S(t)) \subseteq\{z \in \mathbb{C},|z| \leq 1\}
$$

Además, utilizando la hipótesis a) y b) del Teorema de Gearhart se tiene

$$
\left\{\frac{2 \pi n i}{t} ; n \in \mathbb{N}\right\} \subset \rho(A) \text { y } \sup _{n \in \mathbb{N}}\left\|\left(\frac{2 \pi n i}{t} I-A\right)^{-1}\right\| \leq M
$$

para $\lambda=0$, entonces por el Corolario previo, obtenemos que $1 \in \rho(S(t))$. Análogamente procedemos para $\lambda=i \theta$, obteniendo $e^{i \theta} \in \rho(S(t))$ para todo $\theta \in \mathbb{R}$. Por lo tanto, la frontera de la bola unitaria está en el resolvente de $S(t)$.

Así, el espectro de $S(t)$ está contenido en el disco abierto de radio 1, i.e.,

$$
\sigma(S(t)) \subseteq\{z \in \mathbb{C},|z|<1\}
$$

Ahora sabemos que $\sigma(S(t))$ es un conjunto compacto en $\mathbb{C}$, luego

$$
r_{\sigma}(S(t))=\operatorname{máx}_{\lambda \in \sigma(S(t))}|\lambda|=\left|\lambda_{o}\right|<1 \text { para algún } \lambda_{o} \in \sigma(S(t)) .
$$

Tenemos que se verifica: $e^{t w_{o}(A)}=r_{\sigma}(S(t))$, luego $e^{t w_{o}(A)}<1$ y esto nos permite deducir que $t w_{o}(A)<0$.

Así, como $t>0$ entonces $w_{o}(A)<0$, y de la definición de $w_{o}(A)$ tenemos

$$
0>w_{o}(A)=\lim _{z \rightarrow \infty} \frac{\ln \|S(z)\|}{z},
$$

es decir, existe $L>0$ tal que

$$
\lim _{z \rightarrow \infty} \frac{\ln \|S(z)\|}{z}=-L
$$

Entonces, dado $\epsilon>0$, existe $M>0$ tal que si $z>M$ implica $\left|\frac{\ln \|S(z)\|}{z}+L\right|<\epsilon$. Ahora considerando $\epsilon:=\frac{L}{2}$, tenemos $\ln \|S(z)\|<\frac{-L z}{2}$, para $z>M$.

Finalmente tomando la exponencial a esta desigualdad, tenemos $\|S(z)\|<e^{\frac{-L z}{2}}$ para $z>M$, es decir $(S(t))_{t \geq 0}$ es exponencialmente estable.

\section{Comentarios}

En esta sección queremos citar algunas aplicaciones del Teorema de Gearhart, por ejemplo los artículos [4], [5], y enfatizar la riqueza en modelos que posee el libro [1].

Así, el Teorema de Gearhart también se usa para probar la no estabilidad exponencial de algunos sistemas disipativos. 


\section{REFERENCIAS BIBLIOGRÁFICAS}

[1] Liu Z. and Zheng S., (1999). Semigroups associated with dissipative systems. Chapman Hall / CRC.

[2] Pazy A. (1983). Semigroups of linear operators and applications to partial differential equations. Applied Mathematical Sciences. 44 Springer Verlag, Berlin.

[3] Prüs J. (1984). On the spectrum of $C_{0^{-}}$semigroup. Transactions of the American Mathematical Society. $284: 847-857$.

[4] Santiago Y. (2012). Global existence and exponential stability for a coupled wave system. Journal of Mathematical Sciences: Advances and Applications, Vol 16, No 1-2: 29-46.

[5] Santiago Y. (2013). Global existence and Exponential stability for a Timoshenko Beam model. International Journal of Applied Science and Technology. Vol 3, No 7: 141-150. 